\title{
Local and Compositional Reasoning for Optimized Reactive Systems
}

\author{
Mitesh Jain ${ }^{1(\bowtie)}$ and Panagiotis Manolios ${ }^{2}$ \\ 1 Synopsys Inc., Mountain View, USA \\ mitesh.jain@synopsys.com \\ 2 Northeastern University, Boston, USA \\ pete@ccs.neu.edu
}

\begin{abstract}
We develop a compositional, algebraic theory of skipping refinement, as well as local proof methods to effectively analyze the correctness of optimized reactive systems. A verification methodology based on refinement involves showing that any infinite behavior of an optimized low-level implementation is a behavior of the high-level abstract specification. Skipping refinement is a recently introduced notion to reason about the correctness of optimized implementations that run faster than their specifications, i.e., a step in the implementation can skip multiple steps of the specification. For the class of systems that exhibit bounded skipping, existing proof methods have been shown to be amenable to mechanized verification using theorem provers and model-checkers. However, reasoning about the correctness of reactive systems that exhibit unbounded skipping using these proof methods requires reachability analysis, significantly increasing the verification effort. In this paper, we develop two new sound and complete proof methods for skipping refinement. Even in presence of unbounded skipping, these proof methods require only local reasoning and, therefore, are amenable to mechanized verification. We also show that skipping refinement is compositional, so it can be used in a stepwise refinement methodology. Finally, we illustrate the utility of the theory of skipping refinement by proving the correctness of an optimized event processing system.
\end{abstract}

\section{Introduction}

Reasoning about the correctness of a reactive system using refinement involves showing that any (infinite) observable behavior of a low-level, optimized implementation is a behavior allowed by the simple, high-level abstract specification. Several notions of refinement like trace containment, (bi)simulation refinement, stuttering (bi)simulation refinement, and skipping refinement $[4,10,14,20,22]$ have been proposed in the literature to directly account for the difference in the abstraction levels between a specification and an implementation. Two attributes of crucial importance that enable us to effectively verify complex reactive systems using refinement are: (1) Compositionality: this allows us to decompose a monolithic proof establishing that a low-level concrete implementation refines

(C) The Author(s) 2019

I. Dillig and S. Tasiran (Eds.): CAV 2019, LNCS 11561, pp. 553-571, 2019.

https://doi.org/10.1007/978-3-030-25540-4_32 
a high-level abstract specification into a sequence of simpler refinement proofs, where each of the intermediate refinement proof can be performed independently using verification tools best suited for it; (2) Effective proof methods: analyzing the correctness of a reactive system requires global reasoning about its infinite behaviors, a task that is often difficult for verification tools. Hence it is crucial that the refinement-based methodology also admits effective proof methods that are amenable for mechanized reasoning.

It is known that the (bi)simulation refinement and stuttering (bi)simulation refinement are compositional and support the stepwise refinement methodology [20,24]. Moreover, the proof methods associated with them are local, i.e., they only require reasoning about states and their successors. Hence, they are amenable to mechanized reasoning. However, to the best of our knowledge, it is not known if skipping refinement is compositional. Skipping refinement is a recently introduced notion of refinement for verifying the correctness of optimized implementations that can "execute faster" than their simple high-level specifications, i.e., a step in the implementation can skip multiple steps in the specification. Examples of such systems include superscalar processors, concurrent and parallel systems and optimizing compilers. Two proof methods, reduced well-founded skipping simulation and well-founded skipping simulation have been introduced to reason about skipping refinement for the class of systems that exhibit bounded skipping [10]. These proof methods were used to verify the correctness of several systems that otherwise were difficult to automatically verify using current model-checkers and automated theorem provers. However, when skipping is unbounded, the proof methods in [10] require reachability analysis, and therefore are not amenable to automated reasoning. To motivate the need for alternative proof methods for effective reasoning, we consider the event processing system (EPS), discussed in [10].

\subsection{Motivating Example}

An abstract high-level specification, AEPS, of an event processing system is defined as follows. Let $E$ be a set of events and $V$ be a set of state variables. A state of AEPS is a triple $\langle t, S c h, S t\rangle$, where $t$ is a natural number denoting the current time; $S c h$ is a set of pairs $\left\langle e, t_{e}\right\rangle$, where $e \in E$ is an event scheduled to be executed at time $t_{e} \geq t ; S t$ is an assignment to state variables in $V$. The transition relation for the AEPS system is defined as follows. If at time $t$ there is no $\langle e, t\rangle \in S c h$, i.e., there is no event scheduled to be executed at time $t$, then $t$ is incremented by 1 . Otherwise, we (nondeterministically) choose and execute an event of the form $\langle e, t\rangle \in S c h$. The execution of an event may result in modifying $S t$ and also removing and adding a finite number of new pairs $\left\langle e^{\prime}, t^{\prime}\right\rangle$ to $S c h$. We require that $t^{\prime}>t$. Finally, execution involves removing the executed event $\langle e, t\rangle$ from $S c h$. Now consider, tEPS, an optimized implementation of AEPS. As before, a state is a triple $\langle t, S c h, S t\rangle$. However, unlike the abstract system which just increments time by 1 when there are no events scheduled at the current time, the optimized system finds the earliest time in future an event is scheduled to execute. The transition relation of tEPS is defined as follows. An event $\left(e, t_{e}\right)$ 
with the minimum time is selected, $t$ is updated to $t_{e}$ and the event $e$ is executed, as in the AEPS. Consider an execution of AEPS and tEPS in Fig. 1. (We only show the prefix of executions). Suppose at $t=0, S c h$ be $\left\{\left(e_{1}, 0\right)\right\}$. The execution of event $e_{1}$ add a new pair $\left(e_{2}, k\right)$ to $S c h$, where $k$ is a positive integer. AEPS at $t=0$, executes the event $e_{1}$, adds a new pair $\left(e_{2}, k\right)$ to $S c h$, and updates $t$ to 1 . Since no events are scheduled to execute before $t=k$, the AEPS system repeatedly increments $t$ by 1 until $t=k$. At $t=k$, it executes the event $e_{2}$. At time $t=0$, tEPS executes $e_{1}$. The next event is scheduled to execute at time $t=k$; hence it updates in one step $t$ to $k$. Next, in one step it executes the event $e_{2}$. Note that tEPS runs faster than AEPS by skipping over abstract states when no event is scheduled for execution at the current time. If $k>1$, the step from $s_{2}$ to $s_{3}$ in tEPS neither corresponds to stuttering nor to a single step of the AEPS. Therefore notions of refinement based on stuttering simulation and bisimulation cannot be used to show that tEPS refines AEPS.

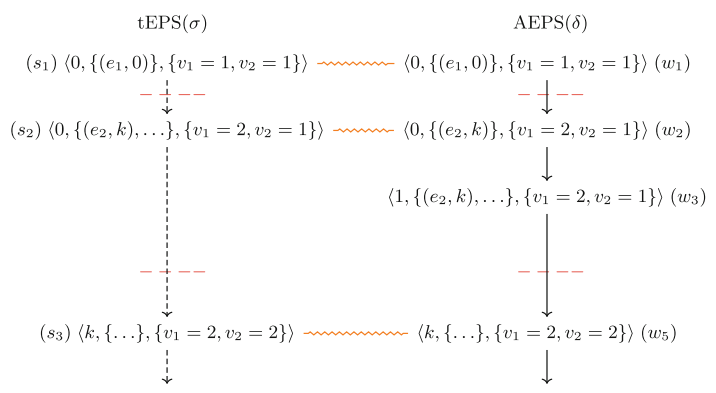

Fig. 1. Event simulation system

It was argued in [10] that skipping refinement is an appropriate notion of correctness that directly accounts for the skipping behavior exhibited by tEPS. Though, tEPS was used to motivate the need for a new notion of refinement, the proof methods proposed in [10] are not effective to prove the correctness of tEPS. This is because, execution of an event in tEPS may add new events that are scheduled to execute at an arbitrary time in future, i.e., in general $k$ in the above example execution is unbounded. Hence, the proof methods in [10] would require unbounded reachability analysis which often is problematic for automated verification tools. Even in the particular case when one can a priori determine an upper bound on $k$ and unroll the transition relation, the proof methods in [10] are viable for mechanical reasoning only if the upper bound $k$ is relatively small.

In this paper, we develop local proof methods to effectively analyze the correctness of optimized reactive systems using skipping refinement. These proof methods reduce global reasoning about infinite computations to local reasoning about states and their successor and are applicable even if the optimized implementation exhibits unbounded skipping. Moreover, we show that the proposed 
proof methods are complete, i.e., if a system $\mathcal{M}_{1}$ is a skipping refinement of $\mathcal{M}_{2}$ under a suitable refinement map, then we can always locally reason about them. We also develop an algebraic theory of skipping refinement. In particular, we show that skipping simulation is closed under relational composition. Thus, skipping refinement aligns with the stepwise refinement methodology. Finally, we illustrate the benefits of the theory of skipping refinement and the associated proof methods by verifying the correctness of optimized event processing systems in ACL2s [3].

\section{Preliminaries}

A transition system model of a reactive system captures the concept of a state, atomic transitions that modify state during the course of a computation, and what is observable in a state. Any system with a well defined operational semantics can be mapped to a labeled transition system.

Definition 1 Labeled Transition System. A labeled transition system (TS) is a structure $\langle S, \rightarrow, L\rangle$, where $S$ is a non-empty (possibly infinite) set of states, $\rightarrow \subseteq S \times S$, is a left-total transition relation (every state has a successor), and $L$ is a labeling function whose domain is $S$.

Notation: We first describe the notational conventions used in the paper. Function application is sometimes denoted by an infix dot "." and is left-associative. The composition of relation $R$ with itself $i$ times (for $0<i \leq \omega$ ) is denoted $R^{i}$ ( $\omega=\mathbb{N}$ and is the first infinite ordinal). Given a relation $R$ and $1<k \leq \omega, R^{<k}$ denotes $\bigcup_{1 \leq i<k} R^{i}$ and $R^{\geq k}$ denotes $\bigcup_{\omega>i \geq k} R^{i}$. Instead of $R^{<\omega}$ we often write the more common $R^{+}$. $\uplus$ denotes the disjoint union operator. Quantified expressions are written as $\langle Q x: r: t\rangle$, where $Q$ is the quantifier (e.g., $\exists, \forall, \min , \bigcup), x$ is a bound variable, $r$ is an expression that denotes the range of variable $x$ (true, if omitted), and $t$ is a term.

Let $\mathcal{M}=\langle S, \rightarrow, L\rangle$ be a transition system. An $\mathcal{M}$-path is a sequence of states such that for adjacent states, $s$ and $u, s \rightarrow u$. The $j^{\text {th }}$ state in an $\mathcal{M}$-path $\sigma$ is denoted by $\sigma . j$. An $\mathcal{M}$-path $\sigma$ starting at state $s$ is a fullpath, denoted by fp.o.s, if it is infinite. An $\mathcal{M}$-segment, $\left\langle v_{1}, \ldots, v_{k}\right\rangle$, where $k \geq 1$ is a finite $\mathcal{M}$-path and is also denoted by $\vec{v}$. The length of an $\mathcal{M}$-segment $\vec{v}$ is denoted by $|\vec{v}|$. Let $I N C$ be the set of strictly increasing sequences of natural numbers starting at 0 . The $i^{t h}$ partition of a fullpath $\sigma$ with respect to $\pi \in I N C$, denoted by ${ }^{\pi} \sigma^{i}$, is given by an $\mathcal{M}$-segment $\langle\sigma(\pi . i), \ldots, \sigma(\pi(i+1)-1)\rangle$.

\section{Theory of Skipping Refinement}

In this section we first briefly recall the notion of skipping simulation as described in [10]. We then study the algebraic properties of skipping simulation and show that a theory of refinement based on it is compositional and therefore can be used in a stepwise refinement based verification methodology. 
The definition of skipping simulation is based on the notion of matching. Informally, a fullpath $\sigma$ matches a fullpath $\delta$ under the relation $B$ iff the fullpaths can be partitioned in to non-empty, finite segments such that all elements in a segment of $\sigma$ are related to the first element in the corresponding segment of $\delta$.

Definition 2 smatch [10]. Let $\mathcal{M}=\langle S, \rightarrow, L\rangle$ be a transition system, $\sigma, \delta$ be fullpaths in $\mathcal{M}$. For $\pi, \xi \in I N C$ and binary relation $B \subseteq S \times S$, we define

$$
\begin{aligned}
& \operatorname{scorr}(B, \sigma, \pi, \delta, \xi) \equiv\left\langle\forall i \in \omega::\left\langle\forall s \in{ }^{\pi} \sigma^{i}:: s B \delta(\xi . i)\right\rangle\right\rangle \text { and } \\
& \operatorname{smatch}(B, \sigma, \delta) \equiv\langle\exists \pi, \xi \in I N C:: \operatorname{scorr}(B, \sigma, \pi, \delta, \xi)\rangle .
\end{aligned}
$$

Figure 1 illustrates the notion of matching using our running example: $\sigma$ is the fullpath of the concrete system and $\delta$ is a fullpath of the absract system. (The figure only shows the prefix of the fullpaths). The other parameter for matching is the relation $B$, which is just the identity function. In order to show that $\operatorname{smatch}(B, \sigma, \delta)$ holds, we have to find $\pi, \xi \in I N C$ satisfying the definition. In Fig. 1, we separate the partitions induced by our choice for $\pi, \xi$ using - - and connect elements related by $B$ with $\sim$. Since all elements of a $\sigma$ partition are related to the first element of the corresponding $\delta$ partition, $\operatorname{scorr}(B, \sigma, \pi, \delta, \xi)$ holds, therefore, $\operatorname{smatch}(B, \sigma, \delta)$ holds.

Using the notion of matching, skipping simulation is defined as follows. Notice that skipping simulation is defined using a single transition system; it is easy to lift the notion defined on a single transition system to one that relates two transition systems by taking the disjoint union of the transition systems.

Definition 3 Skipping Simulation (SKS). $B \subseteq S \times S$ is a skipping simulation on a $T S \mathcal{M}=\langle S, \rightarrow, L\rangle$ iff for all $s, w$ such that $s B w$, both of the following hold.

(SKS1) L.s = L.w

(SKS2) $\langle\forall \sigma: f p . \sigma . s:\langle\exists \delta: f p . \delta . w: \operatorname{smatch}(B, \sigma, \delta)\rangle\rangle$

Theorem 1. Let $\mathcal{M}$ be a TS. If $B$ is a stuttering simulation (STS) on $\mathcal{M}$ then $B$ is an $S K S$ on $\mathcal{M}$.

Proof: Follows directly from the definitions of SKS and STS [18].

\subsection{Algebraic Properties}

We now study the algebraic properties of SKS. We show that it is closed under arbitrary union. We also show that SKS is closed under relational composition. The later property is particularly useful since it enables us to use stepwise refinement and to modularly analyze the correctness of complex systems.

Lemma 1. Let $\mathcal{M}$ be a TS and $\mathcal{C}$ be a set of $S K S$ 's on $\mathcal{M}$. Then $G=\langle\cup B$ : $B \in \mathcal{C}: B\rangle$ is an $S K S$ on $\mathcal{M}$.

Corollary 1. For any $T S \mathcal{M}$, there is a greatest $S K S$ on $\mathcal{M}$. 
Lemma 2. SKS are not closed under negation and intersection.

The following lemma shows that skipping simulation is closed under relational composition.

Lemma 3. Let $\mathcal{M}$ be a TS. If $P$ and $Q$ are SKS's on $\mathcal{M}$, then $R=P ; Q$ is an $S K S$ on $\mathcal{M}$.

Proof: To show that $R$ is an SKS on $\mathcal{M}=\langle S, \rightarrow, L\rangle$, we show that for any $s, w \in S$ such that $s R w$, SKS1 and SKS2 hold. Let $s, w \in S$ and $s R w$. From the definition of $R$, there exists $x \in S$ such that $s P x$ and $x Q w$. Since $P$ and $Q$ are SKS's on $\mathcal{M}$, L.s $=$ L. $x=L . w$, hence, SKS1 holds for $R$.

To prove that SKS2 holds for $R$, consider a fullpath $\sigma$ starting at $s$. Since $P$ and $Q$ are SKSs on $\mathcal{M}$, there is a fullpath $\tau$ in $\mathcal{M}$ starting at $x$, a fullpath $\delta$ in $\mathcal{M}$ starting at $w$ and $\alpha, \beta, \theta, \gamma \in I N C$ such that $\operatorname{scorr}(P, \sigma, \alpha, \tau, \beta)$ and $\operatorname{scorr}(Q, \tau, \theta, \delta, \gamma)$ hold. We use the fullpath $\delta$ as a witness and define $\pi, \xi \in I N C$ such that $\operatorname{scorr}(R, \sigma, \pi, \delta, \xi)$ holds.

We define a function, $r$, that given $i$, corresponding to the index of a partition of $\tau$ under $\beta$, returns the index of the partition of $\tau$ under $\theta$ in which the first element of $\tau^{\prime}$ 's $i^{t h}$ partition under $\beta$ resides. $r . i=j$ iff $\theta . j \leq \beta . i<\theta(j+1)$. Note that $r$ is indeed a function, as every element of $\tau$ resides in exactly one partition of $\theta$. Also, since there is a correspondence between the partitions of $\alpha$ and $\beta$, (by $\operatorname{scorr}(P, \sigma, \alpha, \tau, \beta)$ ), we can apply $r$ to indices of partitions of $\sigma$ under $\alpha$ to find where the first element of the corresponding $\beta$ partition resides. Note that $r$ is non-decreasing: $a<b \Rightarrow r . a \leq r . b$.

We define $\pi \alpha \in I N C$, a strictly increasing sequence that will allow us to merge adjacent partitions in $\alpha$ as needed to define the strictly increasing sequence $\pi$ on $\sigma$ used to prove SKS2. Partitions in $\pi$ will consist of one or more $\alpha$ partitions. Given $i$, corresponding to the index of a partition of $\sigma$ under $\pi$, the function $\pi \alpha$ returns the index of the corresponding partition of $\sigma$ under $\alpha$.

$$
\begin{gathered}
\pi \alpha(0)=0 \\
\pi \alpha(i)=\min j \in \omega \text { s.t. }|\{k: 0<k \leq j \wedge r . k \neq r(k-1)\}|=i
\end{gathered}
$$

Note that $\pi \alpha$ is an increasing function, i.e., $a<b \Rightarrow \pi \alpha(a)<\pi \alpha(b)$. We now define $\pi$ as follows.

$$
\pi . i=\alpha(\pi \alpha . i)
$$

There is an important relationship between $r$ and $\pi \alpha$

$$
r(\pi \alpha . i)=\cdots=r(\pi \alpha(i+1)-1)
$$

That is, for all $\alpha$ partitions that are in the same $\pi$ partition, the initial states of the corresponding $\beta$ partitions are in the same $\theta$ partition.

We define $\xi$ as follows: $\xi . i=\gamma(r(\pi \alpha . i))$. 
We are now ready to prove SKS2. Let $s \in{ }^{\pi} \sigma^{i}$. We show that $s R \delta(\xi . i)$. By the definition of $\pi$, we have

$$
s \in{ }^{\alpha} \sigma^{\pi \alpha \cdot i} \vee \cdots \vee s \in{ }^{\alpha} \sigma^{\pi \alpha(i+1)-1}
$$

Hence,

$$
s P \tau(\beta(\pi \alpha . i)) \vee \cdots \vee s P \tau(\beta(\pi \alpha(i+1)-1))
$$

Note that by the definition of $r$ (apply $r$ to $\pi \alpha . i)$ :

$$
\theta(r(\pi \alpha . i)) \leq \beta(\pi \alpha . i)<\theta(r(\pi \alpha . i)+1)
$$

Hence,

$$
\tau(\beta(\pi \alpha . i)) Q \delta(\gamma(r(\pi \alpha . i))) \vee \cdots \vee \tau(\beta(\pi \alpha(i+1)-1)) Q \delta(\gamma(r(\pi \alpha(i+1)-1)))
$$

By the definition of $\xi$ and the relationship between $r$ and $\pi \alpha$ described above, we simplify the above formula as follows.

$$
\tau(\beta(\pi \alpha . i)) Q \delta(\xi . i) \vee \cdots \vee \tau(\beta(\pi \alpha(i+1)-1)) Q \delta(\xi . i)
$$

Therefore, by the definition of $R$, we have that $s R \delta(\xi . i)$ holds.

Theorem 2. The reflexive transitive closure of an $S K S$ is an $S K S$.

Theorem 3. Given a TS $\mathcal{M}$, the greatest $S K S$ on $\mathcal{M}$ is a preorder.

Proof. Let $G$ be the greatest SKS on $\mathcal{M}$. From Theorem 2, $G^{*}$ is an SKS. Hence $G^{*} \subseteq G$. Furthermore, since $G \subseteq G^{*}$, we have that $G=G^{*}$, i.e., $G$ is reflexive and transitive.

\subsection{Skipping Refinement}

We now recall the notion of skipping refinement [10]. We use skipping simulation, a notion defined in terms of a single transition system, to define skipping refinement, a notion that relates two transition systems: an abstract transition system and a concrete transition system. Informally, if a concrete system is a skipping refinement of an abstract system, then its observable behaviors are also behaviors of the abstract system, modulo skipping (which includes stuttering). The notion is parameterized by a refinement map, a function that maps concrete states to their corresponding abstract states. A refinement map along with a labeling function determines what is observable at a concrete state.

Definition 4 Skipping Refinement. Let $\mathcal{M}_{A}=\left\langle S_{A}, \stackrel{A}{\rightarrow}, L_{A}\right\rangle$ and $\mathcal{M}_{C}=$ $\left\langle S_{C}, \stackrel{C}{\longrightarrow}, L_{C}\right\rangle$ be transition systems and let $r: S_{C} \rightarrow S_{A}$ be a refinement map. We say $\mathcal{M}_{C}$ is a skipping refinement of $\mathcal{M}_{A}$ with respect to $r$, written $\mathcal{M}_{C} \lesssim r \mathcal{M}_{A}$, if there exists a binary relation $B$ such that all of the following hold. 
1. $\left\langle\forall s \in S_{C}:: s B r . s\right\rangle$ and

2. $B$ is an $S K S$ on $\left\langle S_{C} \uplus S_{A}, \stackrel{C}{\longrightarrow} \uplus \stackrel{A}{\rightarrow}, \mathcal{L}\right\rangle$ where $\mathcal{L} . s=L_{A}(s)$ for $s \in S_{A}$, and $\mathcal{L} . s=L_{A}(r . s)$ for $s \in S_{C}$.

Next, we use the property that skipping simulation is closed under relational composition to show that skipping refinement supports modular reasoning using a stepwise refinement approach. In order to verify that a low-level complex implementation $\mathcal{M}_{C}$ refines a simple high-level abstract specification $\mathcal{M}_{A}$ one proceeds as follows: starting with $\mathcal{M}_{A}$ define a sequence of intermediate systems leading to the final complex implementation $\mathcal{M}_{C}$. Any two successive systems in the sequence differ only in relatively few aspects of their behavior. We then show that, at each step in the sequence, the system at the current step is a refinement of the previous one. Since at each step, the verification effort is focused only on the few differences in behavior between two systems under consideration, proof obligations are simpler than the monolithic proof. Note that this methodology is orthogonal to (horizontal) modular reasoning that infers the correctness of a system from the correctness of its sub-components.

Theorem 4. Let $\mathcal{M}_{1}=\left\langle S_{1}, \stackrel{1}{\rightarrow}, L_{1}\right\rangle, \mathcal{M}_{2}=\left\langle S_{2}, \stackrel{2}{\rightarrow}, L_{2}\right\rangle$, and $\mathcal{M}_{3}=\left\langle S_{3}, \stackrel{3}{\rightarrow}, L_{3}\right\rangle$ be TSs, $p: S_{1} \rightarrow S_{2}$ and $r: S_{2} \rightarrow S_{3}$. If $\mathcal{M}_{1} \lesssim p \mathcal{M}_{2}$ and $\mathcal{M}_{2} \lesssim r \mathcal{M}_{3}$, then $\mathcal{M}_{1} \lesssim p ; r \mathcal{M}_{3}$

Proof: Since $\mathcal{M}_{1} \lesssim p \mathcal{M}_{2}$, we have an SKS, say $A$, such that $\left\langle\forall s \in S_{1}:: s A(p . s)\right\rangle$. Furthermore, without loss of generality we can assume that $A \subseteq S_{1} \times S_{2}$. Similarly, since $\mathcal{M}_{2} \lesssim r \mathcal{M}_{3}$, we have an SKS, say $B$, such that $\left\langle\forall s \in S_{2}:: s B(r . s)\right\rangle$ and $B \subseteq S_{2} \times S_{3}$. Define $C=A ; B$. Then we have that $C \subseteq S_{1} \times S_{3}$ and $\left\langle\forall s \in S_{1}:: s C r(p . s)\right\rangle$. Also, from Theorem $2, C$ is an SKS on $\left\langle S_{1} \uplus S_{3}, \stackrel{1}{\rightarrow} \uplus \stackrel{3}{\rightarrow}, \mathcal{L}\right\rangle$, where $\mathcal{L} . s=L_{3}(s)$ if $s \in S_{3}$ else $\mathcal{L} . s=L_{3}(r(p . s))$.

Formally, to establish that a complex low-level implementation $\mathcal{M}_{C}$ refines a simple high-level abstract specification $\mathcal{M}_{A}$, one defines intermediate systems $\mathcal{M}_{1}, \ldots \mathcal{M}_{n}$, where $n \geq 1$ and establishes the following: $\mathcal{M}_{C}=\mathcal{M}_{0} \lesssim r_{0} \mathcal{M}_{1} \lesssim r_{1}$ $\ldots \lesssim r_{n-1} \mathcal{M}_{n}=\mathcal{M}_{A}$. Then from Theorem 4, we have that $\mathcal{M}_{C} \lesssim r \mathcal{M}_{A}$, where $r=r_{0} ; r_{1} ; \ldots ; r_{n-1}$. We illustrate the utility of this approach in Sect. 5 by proving the correctness of an optimized event processing systems.

Theorem 5. Let $\mathcal{M}=\langle S, \rightarrow, L\rangle$ be a TS. Let $\mathcal{M}^{\prime}=\left\langle S^{\prime}, \rightarrow^{\prime}, L^{\prime}\right\rangle$ where $S^{\prime} \subseteq S$, $\rightarrow^{\prime} \subseteq S^{\prime} \times S^{\prime}, \rightarrow^{\prime}$ is a left-total subset of $\rightarrow^{+}$, and $L^{\prime}=\left.L\right|_{S^{\prime}}$. Then $\mathcal{M}^{\prime} \lesssim I \mathcal{M}$, where $I$ is the identity function on $S^{\prime}$.

Corollary 2. Let $\mathcal{M}_{C}=\left\langle S_{C}, \stackrel{C}{\longrightarrow}, L_{C}\right\rangle$ and $\mathcal{M}_{A}=\left\langle S_{A}, \stackrel{A}{\longrightarrow}, L_{A}\right\rangle$ be TSs, $r$ :

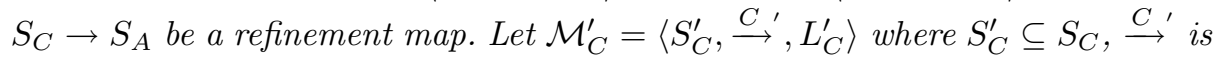
a left-total subset of $\stackrel{C}{\longrightarrow}{ }^{+}$, and $L_{C}^{\prime}=\left.L_{C}\right|_{S_{C}^{\prime}}$. If $\mathcal{M}_{C} \lesssim r \mathcal{M}_{A}$ then $\mathcal{M}_{C}^{\prime} \lesssim_{r^{\prime}} \mathcal{M}_{A}$, where $r^{\prime}$ is $\left.r\right|_{S_{C}^{\prime}}$.

We now illustrate the usefulness of the theory of skipping refinement using our running example of event processing systems. Consider MPEPS, that uses 
a priority queue to find a non-empty set of events (say $E_{t}$ ) scheduled to execute at the current time and executes them. We allow the priority queue in MPEPS to be deterministic or nondeterministic. For example, the priority queue may deterministically select a single event in $E_{t}$ to execute, or based on considerations such as resource utilization it may execute some subset of events in $E_{t}$ in a single step. When reasoning about the correctness of MPEPS, one thing to notice is that there is a difference in the data structures used in the two systems: MPEPS uses a priority queue to effectively find the next set of events to execute in the scheduler, while AEPS uses a simple abstract set representation for the scheduler. Another thing to notice is that MPEPS can "execute faster" than AEPS in two ways: it can increment time by more than 1 and it can execute more than one event in a single step. The theory of skipping refinement developed in this paper enables us to separate out these concerns and apply a stepwise refinement approach to effectively analyse MPEPS.

First, we account for the difference in the data structures between MPEPS and AEPS. Towards this we define an intermediate system MEPS that is identical to MPEPS except that the scheduler in MEPS is now represented as a set of event-time pairs. Under a refinement map, say $p$, that extracts the set of eventtime pairs in the priority queue of MPEPS, a step in MPEPS can be matched by a step in MEPS. Hence, MPEPS $\lesssim_{p}$ MEPS. Next we account for the difference between MEPS and AEPS in the number of events the two systems may execute in a single step. Towards this, observe that the state space of MEPS and tEPS are equal and the transition relation of MEPS is a left-total subset of the transitive closure of the transition relation of tEPS. Hence, from Theorem 5, we infer that MPEPS is a skipping refinement of tEPS using the identity function, say $I_{1}$, as the refinement map, i.e., MEPS $\lesssim I_{1}$ tEPS. Next observe that the state spaces of tEPS and AEPS are equal and the transition relation of tEPS is a left-total subset of the transitive closure of the transition relation of AEPS. Hence, from Theorem 5, tEPS is a skipping refinement of AEPS using the identity function, say $I_{2}$, as the refinement map, i.e., tEPS $\lesssim_{I_{2}}$ AEPS. Finally, from the transitivity of skipping refinement (Theorem 4 ), we conclude that MPEPS $\lesssim p^{\prime}$ AEPS, where $p^{\prime}=p ; I_{1} ; I_{2}$.

\section{Mechanised Reasoning}

To prove that a transition system $\mathcal{M}_{C}$ is a skipping refinement of a transition system $\mathcal{M}_{A}$ using Definition 3, requires us to show that for any fullpath from $\mathcal{M}_{C}$ we can find a matching fullpath from $\mathcal{M}_{A}$. However, reasoning about existence of infinite sequences can be problematic using automated tools. In this section, we develop sound and complete local proof methods that are applicable even if a system exhibits unbounded skipping. We first briefly present the proof methods, reduced well-founded skipping and well-founded skipping simulation, developed in $[10]$.

Definition 5 Reduced Well-founded Skipping [10]. $B \subseteq S \times S$ is a reduced well-founded skipping relation on $T S \mathcal{M}=\langle S, \rightarrow, L\rangle$ iff: 
$(R W F S K 1)\langle\forall s, w \in S: s B w: L . s=L . w\rangle$

(RWFSK2) There exists a function, rankt $: S \times S \rightarrow W$, such that $\langle W, \prec\rangle$ is well-founded and

$$
\begin{aligned}
& \langle\forall s, u, w \in S: s \rightarrow u \wedge s B w: \\
& \quad \text { (a) }(u B w \wedge \operatorname{rankt}(u, w) \prec \operatorname{rankt}(s, w)) \vee \\
& \left.\quad \text { (b) }\left\langle\exists v: w \rightarrow^{+} v: u B v\right\rangle\right\rangle
\end{aligned}
$$

Definition 6 Well-founded Skipping [10]. $B \subseteq S \times S$ is a well-founded skipping relation on $T S \mathcal{M}=\langle S, \rightarrow, L\rangle$ iff:

$(W F S K 1)\langle\forall s, w \in S: s B w: L . s=L . w\rangle$

(WFSK2) There exist functions, rankt $: S \times S \rightarrow W$, rankl $: S \times S \times S \rightarrow \omega$, such that $\langle W, \prec\rangle$ is well-founded and

$$
\begin{aligned}
& \langle\forall s, u, w \in S: s \rightarrow u \wedge s B w: \\
& \quad \text { (a) }\langle\exists v: w \rightarrow v: u B v\rangle \vee \\
& \quad \text { (b) }(u B w \wedge \operatorname{rankt}(u, w) \prec \operatorname{rankt}(s, w)) \vee \\
& \quad \text { (c) }\langle\exists v: w \rightarrow v: s B v \wedge \operatorname{rankl}(v, s, u)<\operatorname{rankl}(w, s, u)\rangle \vee \\
& \text { (d) }\langle\exists v: w \rightarrow \geq 2 v: u B v\rangle\rangle
\end{aligned}
$$

Theorem 6 [10]. Let $\mathcal{M}=\langle S, \rightarrow, L\rangle$ be a TS and $B \subseteq S \times S$. The following statements are equivalent

(i) $B$ is a $S K S$ on $\mathcal{M}$;

(ii) $B$ is a WFSK on $\mathcal{M}$;

(iii) $B$ is a RWFSK on $\mathcal{M}$.

Recall the event processing systems AEPS and tEPS described in Sect.1.1. When no events are scheduled to execute at a given time, say $t$, tEPS increments time $t$ to the earliest time in future, say $k>t$, at which an event is scheduled for execution. Execution of an event can add an event that is scheduled to be executed at an arbitrary time in future. Therefore, we cannot apriori determine an upper-bound on $k$. Using any of the above two proof-methods to reason about skipping refinement would require unbounded reachability analysis (conditions RWFSK2b and WFSK2d), often difficult for automated verification tools. To redress the situation, we develop two new proof methods of SKS; both require only local reasoning about steps and their successors.

Definition 7 Reduced Local Well-founded Skipping. $B \subseteq S \times S$ is a local well-founded skipping relation on $T S \mathcal{M}=\langle S, \rightarrow, L\rangle$ iff:

$(R L W F S K 1)\langle\forall s, w \in S: s B w: L . s=L . w\rangle$

(RLWFSK2) There exist functions, rankt $: S \times S \longrightarrow W$, rankls $: S \times S \longrightarrow \omega$ such that $\langle W, \prec\rangle$ is well founded, and, a binary relation $\mathcal{O} \subseteq S \times S$ 
such that

$$
\begin{aligned}
& \langle\forall s, u, w \in S: s B w \wedge s \rightarrow u: \\
& \quad \text { (a) }(u B w \wedge \operatorname{rankt}(u, w) \prec \operatorname{rankt}(s, w)) \vee \\
& \quad \text { (b) }\langle\exists v: w \rightarrow v: u \mathcal{O} v\rangle\rangle \\
& \text { and } \\
& \langle\forall x, y \in S: x \mathcal{O} y: \\
& \quad \text { (c) } x B y \vee \\
& \quad \text { (d) }\langle\exists z: y \rightarrow z: x \mathcal{O} z \wedge \operatorname{rankls}(z, x)<\operatorname{rankls}(y, x)\rangle\rangle
\end{aligned}
$$

Observe that to prove that a relation is an RLWFSK on a transition system, it is sufficient to reason about single steps of the transition system. Also, note that RLWFSK does not differentiate between skipping and stuttering on the right. This is based on an earlier observation that skipping subsumes stuttering. We used this observation to simplify the definition. However, it can often be useful to differentiate between skipping and stuttering. Next we define local well-founded skipping simulation (LWFSK), a characterization of skipping simulation that separates reasoning about skipping and stuttering on the right (Fig. 2).

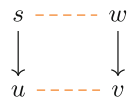

(a)

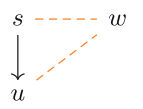

(b)

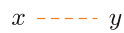

(e)

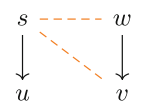

(c)

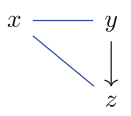

(f)

Fig. 2. Local well-founded skipping simulation (orange line indicates the states are related by $B$ and blue line indicate the states are related by $\mathcal{O}$ ) (Color figure online)

Definition 8 Local Well-founded Skipping. $B \subseteq S \times S$ is a local wellfounded skipping relation on $T S \mathcal{M}=\langle S, \rightarrow, L\rangle$ iff:

$(L W F S K 1)\langle\forall s, w \in S: s B w: L . s=L . w\rangle$

(LWFSK2) There exist functions, rankt $: S \times S \longrightarrow W$, rankl $: S \times S \times S \longrightarrow \omega$, and rankls $: S \times S \longrightarrow \omega$ such that $\langle W, \prec\rangle$ is well founded, and, a 
binary relation $\mathcal{O} \subseteq S \times S$ such that

$$
\begin{aligned}
& \langle\forall s, u, w \in S: s B w \wedge s \rightarrow u: \\
& \quad \text { (a) }\langle\exists v: w \rightarrow v: u B v\rangle \vee \\
& \quad \text { (b) }(u B w \wedge \operatorname{rankt}(u, w) \prec \operatorname{rankt}(s, w)) \vee \\
& \quad \text { (c) }\langle\exists v: w \rightarrow v: s B v \wedge \operatorname{rankl}(v, s, u)<\operatorname{rankl}(w, s, u)\rangle \vee \\
& \quad \text { (d) }\langle\exists v: w \rightarrow v: u \mathcal{O} v\rangle\rangle \\
& \text { and } \\
& \langle\forall x, y \in S: x \mathcal{O} y: \\
& \quad \text { (e) } x B y \vee \\
& \text { (f) }\langle\exists z: y \rightarrow z: x \mathcal{O} z \wedge \operatorname{rankls}(z, x)<\operatorname{rankls}(y, x)\rangle\rangle
\end{aligned}
$$

Like RLWFSK, to prove that a relation is a LWFSK, reasoning about single steps of the transition system suffices. However, LWFSK2b accounts for stuttering on the right, and LWFSK2d along with LWFSK2e and LWFSK2f accounts for skipping on the right. Also observe that states related by $\mathcal{O}$ are not required to be labeled identically and may have no observable relationship to the states related by $B$.

Soundness and Completeness. We next show that RLWFSK and LWFSK in fact completely characterize skipping simulation, i.e., RLWFSK and LWFSK are sound and complete proof rules. Thus if a concrete system $\mathcal{M}_{C}$ is a skipping refinement of $\mathcal{M}_{A}$, one can always effectively reason about it using RLWFSK and LWFSK.

Theorem 7. Let $\mathcal{M}=\langle S, \rightarrow, L\rangle$ be a transition system and $B \subseteq S \times S$. The following statements are equivalent:

(i) $B$ is an $S K S$ on $\mathcal{M}$;

(ii) $B$ is a WFSK on $\mathcal{M}$;

(iii) $B$ is an RWFSK on $\mathcal{M}$;

(iv) $B$ is an RLWFSK on $\mathcal{M}$;

(v) $B$ is a LWFSK on $\mathcal{M}$;

Proof: The equivalence of (i), (ii) and (iii) follows from Theorem 6. That (iv) implies (v) follows from the simple observation that RLWFSK2 implies LWFSK2. To complete the proof, we prove the following two implications. We prove below that (v) implies (ii) in Lemma 4 and that (iii) implies (iv) in Lemma 5.

Lemma 4. If $B$ is a LWFSK on $\mathcal{M}$, then $B$ is a WFSK on $\mathcal{M}$.

Proof. Let $B$ be a LWFSK on $\mathcal{M}$. WFSK1 follows directly from LWFSK1. Let rankt, rankl, and rankls be functions, and $\mathcal{O}$ be a binary relation such that LWFSK2 holds. To show that WFSK2 holds, we use the same rankt and rankl functions and let $s, u, w \in S$ and $s \rightarrow u$ and $s B w$. LWFSK2a, LWFSK2b and 
LWFSK2c are equivalent to WFSK2a, WFSK2b and WFSK2c, respectively, so we show that if only LWFSK2d holds, then WFSK2d holds. Since LWFSK2d holds, there is a successor $v$ of $w$ such that $u \mathcal{O} v$. Since $u \mathcal{O} v$ holds, either LWFSK2e or LWFSK2f must hold between $u$ and $v$. However, since LWFSK2a does not hold, LWFSK2e cannot hold and LWFSK2f must hold, i.e., there exists a successor $v^{\prime}$ of $v$ such that $u \mathcal{O} v^{\prime} \wedge \operatorname{rankls}\left(v^{\prime}, u\right)<\operatorname{rankls}(v, u)$. So, we need a path of at least 2 steps from $w$ to satisfy the universally quantified constraint on $\mathcal{O}$. Let us consider an arbitrary path, $\delta$, such that $\delta .0=w, \delta .1=v$, $\delta .2=v^{\prime}, u \mathcal{O} \delta . i$, LWFSK2e does not hold between $u$ and $\delta . i$ for $i \geq 1$, and $\operatorname{rankls}(\delta .(i+1), u)<\operatorname{rankls}(\delta . i, u)$. Notice that any such path must be finite because rankls is well founded. Hence, $\delta$ is a finite path and there exists a $k \geq 2$ such that LWFSK2e holds between $u$ and $\delta . k$. Therefore, WFSK2d holds, i.e., there is a state in $\delta$ reachable from $w$ in two or more steps which is related to $u$ by $B$.

Lemma 5. If $B$ is RWFSK on $\mathcal{M}$, then $B$ is an RLWFSK on $\mathcal{M}$.

Proof. Let $B$ be an RWFSK on $\mathcal{M}$. RLWFSK1 follows directly from RWFSK1. To show that RLWFSK2 holds, we use any rankt function that can be used to show that RWFSK2 holds. We define $\mathcal{O}$ as follows.

$$
\mathcal{O}=\left\{(u, v):\left\langle\exists z: v \rightarrow^{+} z: u B z\right\rangle\right\}
$$

We define $\operatorname{rankls}(u, v)$ to be the minimal length of a $\mathcal{M}$-segment that starts at $v$ and ends at a state, say $z$, such that $u B z$, if such a segment exists and 0 otherwise. Let $s, u, w \in S, s B w$ and $s \rightarrow u$. If RWFSK2a holds between $s, u$, and $w$, then RLWFSK2a also holds. Next, suppose that RWFSK2a does not hold but RWFSK2b holds, i.e., there is an $\mathcal{M}$-segment $\langle w, a, \ldots, v\rangle$ such that $u B v$; therefore, $u \mathcal{O} a$ and RLWFSK2b holds.

To finish the proof, we show that $\mathcal{O}$ and rankls satisfy the constraints imposed by the second conjunct in RLWFSK2. Let $x, y \in S, x \mathcal{O} y$ and $x \not B \quad y$. From the definition of $\mathcal{O}$, we have that there is an $\mathcal{M}$-segment from $y$ to a state related to $x$ by $B$; let $\vec{y}$ be such a segment of minimal length. From definition of rankls, we have $\operatorname{rankls}(y, x)=|\vec{y}|$. Observe that $y$ cannot be the last state of $\vec{y}$ and $|\vec{y}| \geq 2$. This is because the last state in $\vec{y}$ must be related to $x$ by $B$, but from the assumption we know that $x \not B \quad y$. Let $y^{\prime}$ be a successor of $y$ in $\vec{y}$. Clearly, $x \mathcal{O} y^{\prime}$; therefore, rankls $\left(y^{\prime}, x\right)<|\vec{y}|-1$, since the length of a minimal $\mathcal{M}$-segment from $y^{\prime}$ to a state related to $x$ by $B$, must be less or equal to $|\vec{y}|-1$.

\section{Case Study (Event Processing System)}

In this section, we analyze the correctness of an optimized event processing system (PEPS) that uses a priority queue to find an event scheduled to execute at any given time. We show that PEPS refines AEPS, a simple event processing system described in Sect.1. Our goal is to illustrate the benefits of the theory of skipping refinement and the associated local proof methods developed in the 
paper. We use ACL2s [3], an interactive theorem prover, to define the operational semantics of the systems and mechanize a proof of its correctness.

Operational Semantics of PEPS: A state of PEPS system is a triple $\langle$ tm, otevs, mem $\rangle$, where tm is a natural number denoting current time, otevs is a set of timed-event pairs denoting the scheduler that is ordered with respect to a total order te-< on timed-event pairs, and mem is a collection of variable-integer pairs denoting the shared memory. The transition function of PEPS is defined as follows: if there are no events in otevs, then PEPS just increments the current time by 1 . Otherwise, it picks the first timed-event pair, say $\langle e, t\rangle$ in otevs, executes it and updates the time to $t$. The execution of an event may result in adding new timed-events to the scheduler, removing existing timed-events from the scheduler and updating the memory. Finally, the executed timed-event is removed from the scheduler. This is a simple, generic model of an event processing system. Notice that the ability to remove events can be used to specify systems with preemption [23]: an event scheduled to execute at some future time may be canceled (and possibly rescheduled to be executed at a different time in future) as a result of the execution of an event that preempts it. Notice that, for a given total order, PEPS is a deterministic system.

The execution of an event is modeled using three constrained functions that take as input an event, ev, a time, $t$, and a memory, mem: step-events-add returns the set of new timed-event pairs to add to the scheduler; step-events-rm returns the set of timed-event pairs to remove from the scheduler; and step-memory returns a memory updated as specified by the event. We place minimal constraints on these functions. For example, we only require that step-events-add returns a set of event-time pairs of the form $\left\langle e, t_{e}\right\rangle$ where $t_{e}$ is greater than the current time $t$. The constrained functions are defined using the encapsulate construct in ACL2 and can be instantiated with any executable definitions that satisfy these constraints without affecting the proof of correctness of PEPS. Moreover, note that the particular choice of the total order on timed-event pairs is irrelevant to the proof of correctness of PEPS.

Stepwise Refinement: We show that PEPS refines AEPS using a stepwise refinement approach: first we define an intermediate system HPEPS obtained by augmenting PEPS with history information and show that PEPS is a simulation refinement of HPEPS. Second, we show that HPEPS is a skipping refinement of AEPS. Finally, we appeal to Theorems 1 and 4 to infer that PEPS refines AEPS. Note that the compositionality of skipping refinement enables us to decompose the proof into a sequence of refinement proofs, each of which is simpler. Moreover, the history information in HPEPS is helpful in defining the witnessing binary relation and the rank function required to prove skipping refinement.

An HPEPS state is a four-tuple $\langle$ tm, otevs, mem, $h\rangle$, where tm, otevs, mem are respectively the current time, an ordered set of timed events and a collection of variable-integer pairs, and $\mathrm{h}$ is the history information. The history information $\mathrm{h}$ consists of a Boolean variable valid, time tm, and an ordered set of timed-event pairs otevs and the memory mem. Intuitively, $\mathrm{h}$ records the state preceding the 
current state. The transition function HPEPS is same as the transition function of PEPS except that HPEPS also records the history in $h$.

PEPS Refines HPEPS: Observe that, modulo the history information, a step of PEPS directly corresponds to a step of HPEPS, i.e., PEPS is a bisimulation refinement of HPEPS under a refinement map that projects a PEPS state $\langle$ tm, otevs, mem $\rangle$ to the HPEPS state $\langle$ tm, otevs, mem, $h\rangle$ where the valid component of $\mathrm{h}$ is set to false. But we only prove that it is a simulation refinement, because, from Theorem 1, it suffices to establish that PEPS is a skipping refinement of HPEPS. The proofs primarily require showing that two sets of ordered timed-events that are set equivalent are in fact equal and that adding and removing equivalent sets of timed-event from equal schedulers results in equal schedulers.

HPEPS Refines AEPS: Next we show that HPEPS is a skipping refinement of AEPS under the refinement map $R$, a function that simply projects an HPEPS state to an AEPS state. To show that HPEPS is a skipping refinement of AEPS under the refinement map $R$, from Definition 4 , we must show as witness a binary relation $B$ that satisfies the two conditions. Let $B=\{(s, R . s)$ : $s$ is an HPEPS state $\}$. To establish that $B$ is an SKS on the disjoint union of HPEPS and AEPS, we have a choice of four proof-methods (Sect. 4). Recall that execution of an event can add a new event scheduled to be executed at an arbitrary time in the future. As a result, if we were to use WFSK or RWFSK, the proof obligations from conditions WFSK2d (Definition 5) and RWFSK2b (Definition 6) would require unbounded reachability analysis, something that typically places a big burden on verification tools and their users. In contrast, the proof obligations to establish RLWFSK are local and only require reasoning about states and their successors, which significantly reduces the proof complexity.

RLWFSK1 holds trivially. To prove that RLWFSK2 holds we define a binary relation $\mathcal{O}$ and a rank function rankls and show that they satisfy the two universally quantified formulas in RLWFSK2. Moreover, since HPEPS does not stutter we ignore RLWFSK2a, and that is why we do not define rankt. Finally, our proof obligation is: for all HPEPS $s, u$ and AEPS state $w$ such that $s \rightarrow u$ and $s B w$ holds, there exists a AEPS state $v$ such that $w \rightarrow v$ and $u \mathcal{O} v$ holds.

Verification Effort: We used the defdata framework in ACL2s, to specify the data definitions for the three systems and the definec construct to introduce function definitions along with their input-contracts (pre-conditions) and output-contracts (post-conditions). In addition to admitting a data definition, defdata proves several theorems about the functions that are extremely helpful in automatically discharging type-like proof obligations. We also developed a library to concisely describe functions using higher-order constructs like map and reduce, which made some of the definitions clearer. ACL2s supports first-order quantifiers via the defun-sk construct, which essentially amounts to the use of Hilbert's choice operator. We use defun-sk to model the transition relation for AEPS (a non-deterministic system) and to specify the proof obligations for proving that HPEPS refines AEPS. However, support for automated reasoning 
about quantifiers is limited in ACL2. Therefore, we use the domain knowledge, when possible (e.g., a system is deterministic), to eliminate quantifiers in the proof obligations and provide explicit witnesses for existential quantifiers.

The proof makes essential use of several libraries available in ACL2 for reasoning about lists and sets. In addition, we prove a collection of additional lemmas that can be roughly categorized into four categories. First, we have a collection of lemmas to prove the input-output contracts of the functions. Second, we have a collection of lemmas to show that operations on the schedulers in the three systems preserve various invariants, e.g., that any timed-event in the scheduler is scheduled to execute at a time greater or equal to the current time. Third, we have a collection of lemmas to show that inserting and removing two equivalent sets of timed-events from a scheduler results in an equivalent scheduler. And fourth, we have a collection of lemmas to show that two schedulers are equivalent iff they are set equal. The above lemmas are used to establish a relationship between priority queues, a data structure used by the implementation system, and sets, the corresponding data structure used in the specification system. The behavioral difference between the two systems is accounted for by the notion of skipping refinement. This separation significantly eases understanding as well as mechanical reasoning about the correctness of reactive systems. We have 8 top-level proof obligations and a few dozen supporting lemmas. The entire proof takes about $120 \mathrm{~s}$ on a machine with $2.2 \mathrm{GHz}$ Intel Core i7 with $16 \mathrm{~GB}$ main memory.

\section{Related Work}

Several notions of correctness have been proposed in the literature and their properties been widely studied $[2,5,11,16,17]$. In this paper, we develop a theory of skipping refinement to effectively prove the correctness of optimized reactive systems using automated verification tools. These results establish skipping refinement on par with notions of refinement based on (bi)simulation [22] and stuttering (bi)simulation [20,24], in the sense that skipping refinement is (1) compositional and (2) admits local proofs methods. Together the two properties have been instrumental in significantly reducing the proof complexity in verification of large and complex systems. We developed the theory of skipping refinement using a generic model of transition systems and place no restrictions on the state space size or the branching factor of the transition system. Any system with a well-defined operational semantics can be mapped to a labeled transition system. Moreover, the local proof methods are sound and complete, i.e., if an implementation is a skipping refinement of the specification, we can always use the local proof methods to effectively reason about it.

Refinement-based methodologies have been successfully used to verify the correctness of several realistic hardware and software systems. In [13], several complex concurrent programs were verified using a stepwise refinement methodology. In addition, Kragl and Qadeer [13] also develop a compact representation to facilitate the description of programs at different levels of abstraction and associated refinement proofs. Several back-end compiler transformations are proved 
correct in Compcert [15] using simulation refinement. In [25], several compiler transformations were verified using stuttering refinement and associated local proof methods. Recently, refinement-based methodology has also been applied to verify the correctness of practical distributed systems [8] and a generalpurpose operating system microkernel [12]. The full verification of CertiKOS $[6,7]$, an OS kernel, is based on the notion of simulation refinement. Refinement based approaches have also been extensively used to verify microprocessor designs $[1,9,19,21,26]$. Skipping refinement was used to verify the correctness of optimized memory controllers and a JVM-inspired stack machine [10].

\section{Conclusion and Future Work}

In this paper, we developed the theory of skipping refinement. Skipping refinement is designed to reason about the correctness of optimized reactive systems, a class of systems where a single transition in a concrete low-level implementation may correspond to a sequence of observable steps in the corresponding abstract high-level specification. Examples of such systems include optimizing compilers, concurrent and parallel systems and superscalar processors. We developed sound and complete proof methods that reduce global reasoning about infinite computations of such systems to local reasoning about states and their successors. We also showed that the skipping simulation is closed under composition and therefore is amenable to modular reasoning using a stepwise refinement approach. We experimentally validated our results by analyzing the correctness of an optimized event-processing system in ACL2s. For future work, we plan to precisely classify temporal logic properties that are preserved by skipping refinement. This would enable us to transfer temporal properties from specifications to implementations, after establishing refinement.

\section{References}

1. Aagaard, M.D., Cook, B., Day, N.A., Jones, R.B.: A framework for microprocessor correctness statements. In: Margaria, T., Melham, T. (eds.) CHARME 2001. LNCS, vol. 2144, pp. 433-448. Springer, Heidelberg (2001). https://doi.org/10. 1007/3-540-44798-9_33

2. Basten, T.: Branching bisimilarity is an equivalence indeed!. Inf. Process. Lett. 58, 141-147 (1996)

3. Chamarthi, H.R., Dillinger, P., Manolios, P., Vroon, D.: The ACL2 sedan theorem proving system. In: Abdulla, P.A., Leino, K.R.M. (eds.) TACAS 2011. LNCS, vol. 6605, pp. 291-295. Springer, Heidelberg (2011). https://doi.org/10.1007/978-3642-19835-9_27

4. Clarke, E.M., Grumberg, O., Browne, M.C.: Reasoning about networks with many identical finite-state processes. In: PODC (1986)

5. van Glabbeek, R.J.: The linear time-branching time spectrum (extended abstract). In: Baeten, J.C.M., Klop, J.W. (eds.) CONCUR 1990. LNCS, vol. 458, pp. 278-297. Springer, Heidelberg (1990). https://doi.org/10.1007/BFb0039066 
6. Gu, L., Vaynberg, A., Ford, B., Shao, Z., Costanzo, D.: CertiKOS: a certified kernel for secure cloud computing. In: APSys (2011)

7. Gu, R., et al.: Deep specifications and certified abstraction layers. In: POPL (2015)

8. Hawblitzel, C., et al.: IronFleet: Proving practical distributed systems correct. In: SOSP (2015)

9. Hosabettu, R., Gopalakrishnan, G., Srivas, M.: Formal verification of a complex pipelined processor. Form. Methods Syst. Des. 23, 171-213 (2003)

10. Jain, M., Manolios, P.: Skipping refinement. In: Kroening, D., Păsăreanu, C.S. (eds.) CAV 2015. LNCS, vol. 9206, pp. 103-119. Springer, Cham (2015). https:// doi.org/10.1007/978-3-319-21690-4_7

11. Klarlund, N.: Progress measures and finite arguments for infinite computations. Ph.D. thesis (1990)

12. Klein, G., Sewell, T., Winwood, S.: Refinement in the formal verification of the seL4 microkernel. In: Hardin, D. (ed.) Design and Verification of Microprocessor Systems for High-Assurance Applications, pp. 323-339. Springer, Boston (2010). https://doi.org/10.1007/978-1-4419-1539-9_11

13. Kragl, B., Qadeer, S.: Layered concurrent programs. In: Chockler, H., Weissenbacher, G. (eds.) CAV 2018. LNCS, vol. 10981, pp. 79-102. Springer, Cham (2018). https://doi.org/10.1007/978-3-319-96145-3_5

14. Lamport, L.: What good is temporal logic. Information processing (1993)

15. Leroy, X., Blazy, S.: Formal verification of a c-like memory model and its uses for verifying program transformations. J. Autom. Reason. 41, 1-31 (2008)

16. Liu, X., Yu, T., Zhang, W.: Analyzing divergence in bisimulation semantics. In: POPL (2017)

17. Lynch, N.A., Vaandrager, F.W.: Forward and backward simulations: I. Untimed systems. Inf. Comput. (1995)

18. Manolios, P.: Mechanical verification of reactive systems. Ph.D. thesis, University of Texas (2001)

19. Manolios, P.: Correctness of pipelined machines. In: Hunt, W.A., Johnson, S.D. (eds.) FMCAD 2000. LNCS, vol. 1954, pp. 181-198. Springer, Heidelberg (2000). https://doi.org/10.1007/3-540-40922-X_11

20. Manolios, P.: A compositional theory of refinement for branching time. In: Geist, D., Tronci, E. (eds.) CHARME 2003. LNCS, vol. 2860, pp. 304-318. Springer, Heidelberg (2003). https://doi.org/10.1007/978-3-540-39724-3_28

21. Manolios, P., Srinivasan, S.K.: A complete compositional reasoning framework for the efficient verification of pipelined machines. In: ICCAD (2005)

22. Milner, R.: An algebraic definition of simulation between programs. In: Proceedings of the 2nd International Joint Conference on Artificial Intelligence (1971)

23. Misra, J.: Distributed discrete-event simulation. ACM Comput. Surv. 18, 39-65 (1986)

24. Namjoshi, K.S.: A simple characterization of stuttering bisimulation. In: Ramesh, S., Sivakumar, G. (eds.) FSTTCS 1997. LNCS, vol. 1346, pp. 284-296. Springer, Heidelberg (1997). https://doi.org/10.1007/BFb0058037

25. Namjoshi, K.S., Zuck, L.D.: Witnessing program transformations. In: Logozzo, F., Fähndrich, M. (eds.) SAS 2013. LNCS, vol. 7935, pp. 304-323. Springer, Heidelberg (2013). https://doi.org/10.1007/978-3-642-38856-9_17

26. Ray, S., Jr Hunt, W.A.: Deductive verification of pipelined machines using firstorder quantification. In: Alur, R., Peled, D.A. (eds.) CAV 2004. LNCS, vol. 3114, pp. 31-43. Springer, Heidelberg (2004). https://doi.org/10.1007/978-3-540-278139_3 
Open Access This chapter is licensed under the terms of the Creative Commons Attribution 4.0 International License (http://creativecommons.org/licenses/by/4.0/), which permits use, sharing, adaptation, distribution and reproduction in any medium or format, as long as you give appropriate credit to the original author(s) and the source, provide a link to the Creative Commons license and indicate if changes were made.

The images or other third party material in this chapter are included in the chapter's Creative Commons license, unless indicated otherwise in a credit line to the material. If material is not included in the chapter's Creative Commons license and your intended use is not permitted by statutory regulation or exceeds the permitted use, you will need to obtain permission directly from the copyright holder. 